\title{
OPTICAL MEASUREMENTS FOR CANGAROO-III
}

\author{
R. KIUCHI, M. YUASA, M. OHISHI, A. KAWACHI, M. MORI AND THE \\ CANGAROO COLLABORATION \\ High Energy Cosmic Ray Division, ICRR, University of Tokyo, \\ 5-1-5 Kashiwanoha, Chiba, 277-8582, Japan \\ E-mail:kiuchi@icrr.u-tokyo.ac.jp
}

CANGAROO-III consists of four telescopes installed near Woomera, South Australia to observe celestial gamma-ray sources by detecting Cherenkov light from air showers. Stereo observations have been performed since March 2004 with an improved angular resolution and a lower energy threshold. In this paper, we present some preliminary results from optical measurements with a cooled CCD camera on the reflectivity of the telescope reflector and the atmospheric transmittance.

\section{Introduction}

In October 2005, extensive maintenance work was carried out on the telescopes, including washing the mirror segments. In this period, we measured the reflectivity of the reflectors and the typical atmospheric transmittance at our site. For all measurements, we used a cooled CCD camera (ST$7 \mathrm{XMEi}, \mathrm{SBIG}^{1}$ ) together with a camera lens (F1.4, focal length $50 \mathrm{~mm}$ ) and Johnson-type optical filters to study the dependence on wavelength. The CCD chip (KAF-0402ME, KODAK ${ }^{2}$ ) has high quantum efficiency especially in the shorter waveband which makes it possible to do optical measurements with a U-band filter. We used three optical filters, V-band, B-band, and U-band. The central wavelength of transmittance is $533 \mathrm{~nm}$ (V-band), $434 \mathrm{~nm}$ (B-band), and $362 \mathrm{~nm}$ (U-band).

\section{Reflectivity of the telescope reflector}

The CANGAROO-III reflector is a tessellated parabola with a diameter of $10 \mathrm{~m}$, consisting of 114 spherical mirror segments each with a diameter of $78 \mathrm{~cm}^{3}$. As described above, we washed all segments with water. The reflectivity of the telescope reflectors was measured before and after washing to determine how much the reflectivity had improved. There are several 
methods to evaluate the reflectivity, but we used a cooled CCD camera since this method can estimate the reflectivity of the whole reflector very simply. The procedure was as follows: First, the telescope tracked a bright star and we took a reflected star image on the white screen ${ }^{4}$ using the CCD camera (Fig. 1). Second, the tracking coordinates of the telescope were slightly changed so that the star came into the field of view of the CCD camera, and we took the direct star image (Fig. 2). The direct and the reflected star images were taken with as short an interval as possible so as to minimize the effect of the difference of the sky condition. This procedure was repeated for several stars with each optical filter, and all three optical filters $(\mathrm{V}, \mathrm{B}, \mathrm{U})$ were used with each telescope. These measurements were made for the three telescopes of the CANGAROO-III stereo system (i.e., currently excluding telescope T1) to compare the relative reflectivity among the telescopes. The quantity of the flux of the reflected star image (in CCD counts) divided by that of the direct star image is proportional to the reflectivity of the whole reflector, so we compare the reflectivities using above quantities calculated from the data which were taken before and after the washing of the segments.

Fig. 3 shows the results for telescope 4, and only results of V-and B-band are shown because we do not have U-band data taken before washing. From the results for all three telescopes, we estimate that the reflectivity of the reflector after washing became about 1.3 times greater than that before washing.

\section{Atmospheric transmittance}

The atmospheric transmittance at our site is an important factor to estimate the $\gamma$-ray energy and flux from the observed Cherenkov light. The observation site is located in the desert, and we can assume that the atmo-

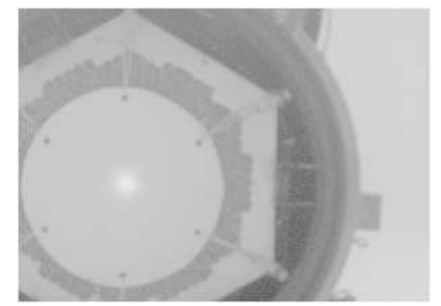

Figure 1. A reflected star image on the white screen taken by a cooled CCD camera.

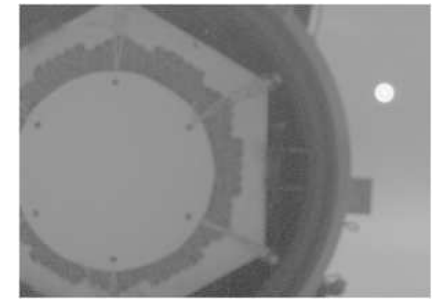

Figure 2. A direct star image. The star is the same in Fig1. 
spheric transmittance is close to that including only Rayleigh scattering. In October 2005, we measured the atmospheric transmittance using bright stars. The concept of these measurements were as follows: By using the fact that the measured flux of a star changes according to its zenith angle, which is the effect of the atmospheric extinction scattering by air molecules and dust, etc., we can estimate the flux of the star above the atmosphere where the scattering is zero by extrapolation from the flux of stars measured at various zenith angles. We can thus calculate the atmospheric transmittance from observed star fluxes. The set-up of the measurement was the same as that used in reflectivity measurements except for the white screen, because this measurement used only direct star images (Fig. 2), and the images of a handful of bright stars at various zenith angles were taken. Fig. 4 is a plot of the atmospheric extinction effect on the flux of stars. Here the airmass represents the thickness of the atmosphere and the definition is $\frac{1}{\cos (\theta)}$, where $\theta$ is the zenith angle.

As shown in Fig. 4, we fit the results with a linear function. From the value of the flux of a star at the point that airmass equals to zero, we estimated the atmospheric transmittance for each measured points (Fig. 5). We used the atmospheric transmittance simulation code, Modtran ${ }^{5}$, to compare our measurements and the "desert aerosol model" in Modtran, which is a model including little aerosol content in the atmosphere, and which is plotted to-

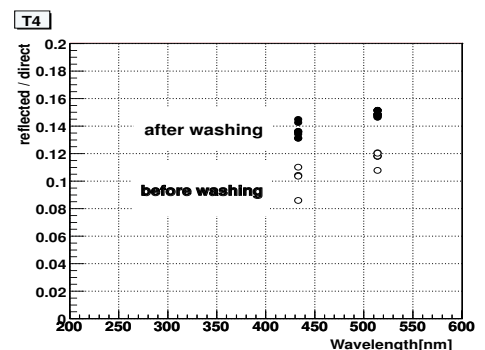

Figure 3. The relative reflectivity of the telescope 4 reflector. Before (open circles) and after (filled circles) the washing works . The vertical axis represents the quantity of the flux of the reflected star divided by that of the direct star. Since there are no data with U-band filter for before the washing works, only data with $\mathrm{V}, \mathrm{B}$ band filter are plotted.

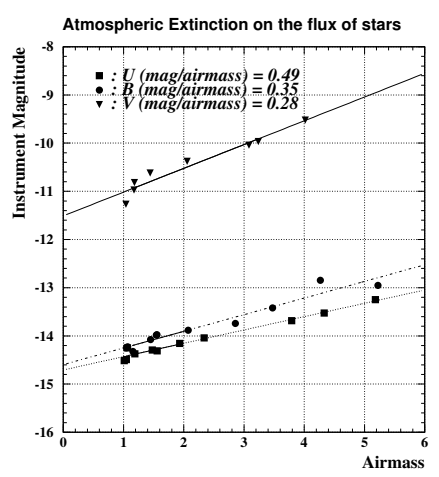

Figure 4. Flux of stars (in magnitude) versus the thickness of the atmosphere (in airmass). The origin of the vertical axis is arbitrary, and the fitted lines to the measured points are also plotted. 
gether with measured points.

Considering the systematic errors such as the calculation of extrapolation and the changing the flux of stars due to instability in sky conditions, we can say that the "desert aerosol model" is similar to the atmosphere at our site.

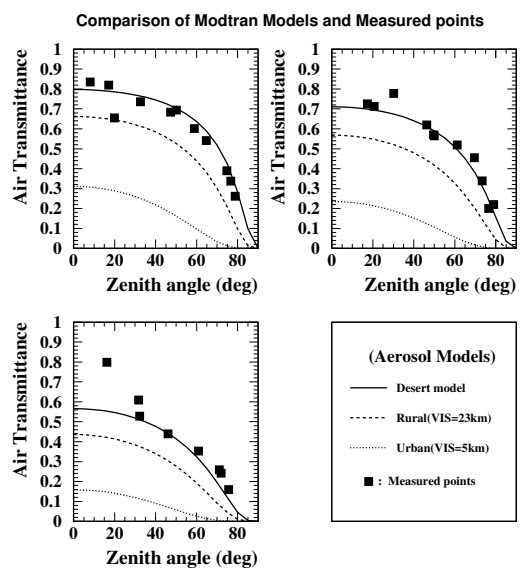

Figure 5. The calculated atmospheric transmittance. The square points are from measurement and the lines are from "desert aerosol model" in Modtran simulation

\section{Summary}

We have carried out optical measurements for CANGAROO-III in October 2005. From the measurements of the reflectivity of the telescope reflectors, the reflectivity has been increased by a factor of 1.3 by washing the mirror segments. Measurements of the atmospheric transmittance indicate the "desert aerosol model" in Modtran simulation is close to our environment.

\section{References}

1. http://www.sbig.com/

2. http://www.kodak.com/global/en/digital/ccd/products/fullframe/KAF0402E/indexKAF-0402E.jhtml

3. Kawachi, A. et.al., Astropart. Phys. 13, 261-269 (2001)

4. Kiuchi, R. et al., Proc. 29th ICRC (Pune), 5, 315 (2005)

5. Berk, A., Bernstein, L.S., Robertson, D.C., 'MODTRAN: A Moderate Resolution Model for LOWTRAN 7', GL-TR-89-0122, 1989 